\title{
Idiopathic Membranous Nephropathy: Diagnostic and Therapeutic Challenges
}

\author{
Amr El-Husseini ${ }^{a}$ d David Saxon $^{a}$ Stuart Jennings ${ }^{a}$ Virgilius Cornea $^{b}$ \\ Laurence Beck $^{c}$ B. Peter Sawaya ${ }^{a}$ \\ ${ }^{a}$ Division of Nephrology and ${ }^{b}$ Department of Pathology, University of Kentucky, Lexington, Ky., and ${ }^{\mathrm{C} D i v i s i o n}$ of \\ Nephrology, Boston University Medical Center, Boston, Mass., USA; ${ }^{\mathrm{d}}$ Mansoura University, Mansoura, Egypt
}

\section{Key Words}

Membranous nephropathy $\cdot$ Human immunodeficiency virus · Adrenocorticotropic hormone

\begin{abstract}
Background: In adults, membranous nephropathy (MN) is a major cause of nephrotic syndrome. While a majority of cases of $\mathrm{MN}$ are idiopathic, secondary forms can be seen in the setting of autoimmune disease, neoplasia, infection, and after being exposed to certain therapeutic agents. Both human immunodeficiency virus (HIV) and hepatitis $C$ virus (HCV) infections could cause MN. However, the effect of coexisting HIV and HCV infection on the spectrum and progression of kidney disease as well as the effect of MN treatment on HIV and HCV infection are not well known. Methods: In this study, we describe a patient with hemophilia A and acquired HIV and HCV infections, a patient who developed severe nephrotic syndrome and for whom renal biopsy showed MN. Results: Patient responded well to adrenocorticotropic hormone gel without adversely affecting HIV or HCV infections. Conclusion: Adrenocorticotropic hormone gel might be useful in the management of complex cases of idiopathic MN.

(c) 2016 S. Karger AG, Basel
\end{abstract}

\section{Introduction}

The association between human immunodeficiency virus (HIV) and renal glomerular disease has been well recognized [1-9]. Significant focus in literature has been placed on HIV-associated nephropathy (HIVAN). HIVAN has been reported as the most common cause of kidney disease in patients with HIV, and it must be considered in all HIV positive patients presenting with proteinuria [1-4]. However, biopsy studies of patients with HIV and kidney disease have found substantial prevalence of other glomerular diseases, including membranous nephropathy (MN) [1,5-9]. MN is a glomerular pathology characterized microscopically by subepithelial immune complex deposits and glomerular basement membrane (GBM) thickening without hypercellularity [10]. The idiopathic subtype of MN has been closely associated with positive tests for anti-phospholipase A2 receptor (PLA2R) antibodies [11-14]. To our knowledge, there are no reports in the literature of patients with wellcontrolled HIV who developed MN without an underlying etiology for secondary $\mathrm{MN}$ and tested positive for anti-PLA2R antibody to indicate idiopathic disease. In this study, we present a case of an HIV-positive patient with anti-PLA2R antibody-associated MN effectively treated with ACTH immunosuppression. 


\section{Case Report}

A 36-year-old, asymptomatic, Caucasian male with a history of hemophilia A, HIV secondary to blood transfusion in childhood and cleared hepatitis $C$ infection was incidentally found on urinalysis to have proteinuria. The patient had normal serum creatinine of $0.74 \mathrm{mg} / \mathrm{dl}$, serum albumin of $2.4 \mathrm{~g} / \mathrm{dl}$ and nephrotic range proteinuria of approximately $7 \mathrm{~g} / 24 \mathrm{~h}$ without peripheral edema on exam. Urinalysis showed $6 \mathrm{RBC} / \mathrm{hpf}$ without cellular casts. Repeated random urine protein/creatinine (UP/C) ratio was ranging between 6 and $8 \mathrm{~g} / \mathrm{g}$ and he had normal complement, and negative anti-nuclear, anti-double stranded DNA, anti-neutrophil cytoplasmic, anti-myeloperoxidase, anti-proteinase 3 antibodies. Tests for hepatitis $C$ revealed the presence of positive antibodies to hepatitis $\mathrm{C}$ with negative hepatitis $\mathrm{C}$ RNA by PCR. HIV viral load was also undetectable with CD4 count of $1,234 / \mu l$. He had been treated with abacavir/lamivudine/zidovudine (Trizivir) for more than 10 years with excellent long-term control. During the workup of his proteinuria, highly active antiretroviral therapy (HAART) regimen was switched to efavirenz/emtricitabine/tenofovir (Atripla) for simplification and then to abacavir/lamivudine/efavirenz to avoid insult to kidneys. Quantitative free kappa and lambda light chains were within normal limits in the normal ratio. Serum protein electrophoresis with immunofixation showed no monoclonal proteins. Kidney ultrasound revealed no abnormalities.

The patient was started on lisinopril and maximized up to $20 \mathrm{mg}$ twice daily as tolerated by the blood pressure, and proteinuria was consistently above $6 \mathrm{~g}$ daily for 6 months. The patient was admitted to the hospital for trans-jugular kidney biopsy with continuous infusion of factor VIII and monitoring of factor VIII activity levels as he had a baseline activity level of $4 \%$. The biopsy was performed without complications.

Biopsy results revealed global glomerulosclerosis involving 2/42 glomeruli (4.7\%). There were normal-sized glomeruli with normal to slightly increased cellularity and no glomerular proliferative lesions, cellular crescents, GBM double contours, or breaks on light microscopy (fig. 1a). Silver stain highlighted GBM spikes (fig. 1b). There was mild tubular atrophy and minimal focal lymphocytic inflammatory infiltrate. Trichrome stain highlighted mild interstitial fibrosis with moderate arterial fibro-intimal thickening. Immunofluorescence microscopy demonstrated $2+$ granular staining of the GBM for IgG, kappa and lambda light chain, and trace granular staining for C3. Linear nonspecific staining in the GBM for albumin was also seen (fig. 1c). Electron microscopy revealed GBM thickening, diffuse effacement of the foot processes, and numerous subepithelial electron dense deposits of complex immune type, partially surrounded by new GBM material formation (fig. 1d). These findings are consistent with stage II $\mathrm{MN}$.

Workup was initiated for causes of secondary MN. CT of chest, abdomen, and pelvis was unremarkable. Colonoscopy revealed no masses or polyps. Prostate-specific antigen and creatinine kinase levels were within normal limits. Serologic tests for hepatitis B, chlamydia, gonorrhea, and syphilis were negative. The patient tested weakly positive for anti-PLA2R antibodies. Because of negative hepatitis $\mathrm{C}$ virus (HCV)-RNA, undetectable HIV viral load and absence of any evidence of secondary $\mathrm{MN}$ in renal biopsy, we considered his $\mathrm{MN}$ as likely to be idiopathic.

Therapy was initiated with twice weekly $80 \mathrm{U}$ injections of adrenocorticotropic hormone (Acthar) gel. UP/C was $6.0 \mathrm{~g} / \mathrm{g}$ when therapy was initiated. After the therapy was completed, UP/C was down to $4.0 \mathrm{~g} / \mathrm{g}$ after 2 months, $0.9 \mathrm{~g} / \mathrm{g}$ at 8 months, $0.5 \mathrm{~g} / \mathrm{g}$ at 12 months and $0.4 \mathrm{~g} / \mathrm{g}$ at 18 months of follow-up. Acthar gel was decreased to once weekly injections after 8 months and then stopped after 16 months of starting therapy (fig. 2). The patient was maintained on lisinopril throughout this time. The patient has remained asymptomatic with regard to kidney disease and additionally, his CD4 count was around 2,000/ $\mathrm{ll}$; also, he had undetectable HIV and HCV viral load for 18 months of follow-up. Repeated western blot after completion of the Acthar gel therapy showed significantly decreased PLA2R antibody (fig. 3) and negative anti-thrombospondin type-1 domain-containing 7A (THSD7A).

Detection of Anti-PLA2R and THSD7A Antibodies by Western Blotting

Human glomerular extract (HGE) and extracts of HKE293 cells transfected with either recombinant human PLA2R or human THSD7A were electrophoresed on SDS-PAGE 4-15\% gradient gels, transferred to nitrocellulose membrane, and western blotted overnight with human serum (at a 1:10 dilution for this case, or 1:25 dilution for a positive control serum with a high titer of antiPLA2R antibodies). The blot was then incubated with sheep antihuman IgG4 antibodies (1:3,000; The Binding Site) followed by peroxidase-conjugated donkey anti-sheep IgG (1:10,000; Jackson ImmunoResearch). After exposure to a chemiluminescent substrate, the blots were exposed to film for 10, 30 and $120 \mathrm{~s}$ and developed. Blots were scanned on a CanoScan Lide 25 scanner and the image assembled using PhotoShop CS4 for Windows.

\section{Discussion}

$\mathrm{MN}$ is a common form of glomerular disease presenting with proteinuria. A majority of patients develop nephrotic syndrome and a significant number go on to develop the end-stage renal disease [15]. MN can be subdivided into idiopathic, without an identifiable etiology, and secondary types with idiopathic being more common [10]. Secondary MN has been associated with chronic infections, drugs, autoimmune disease, and malignancies, and therapy focuses on treating or removing the underlying cause [16-18]. In contrast, treatment of idiopathic $\mathrm{MN}$ is more poorly defined. Polanco et al. [19] reported that $31.7 \%$ of patients with idiopathic MN will see spontaneous remission of proteinuria when treated conservatively with a majority of spontaneous remission occurring within the first 2 years. In this study, spontaneous remission was more common among patients with lower levels of baseline proteinuria, and a gradual decrease to $<50 \%$ of baseline proteinuria within the first 12 months predictive of spontaneous remission. Patients with severe proteinuria, persistent proteinuria, or reduced kidney function are typically treated with immunosuppressive therapy and this therapy typically involves immunosuppression
66

Am J Nephrol 2016;43:65-70

DOI: $10.1159 / 000444593$
El-Husseini/Saxon/Jennings/Cornea/ Beck/Sawaya 

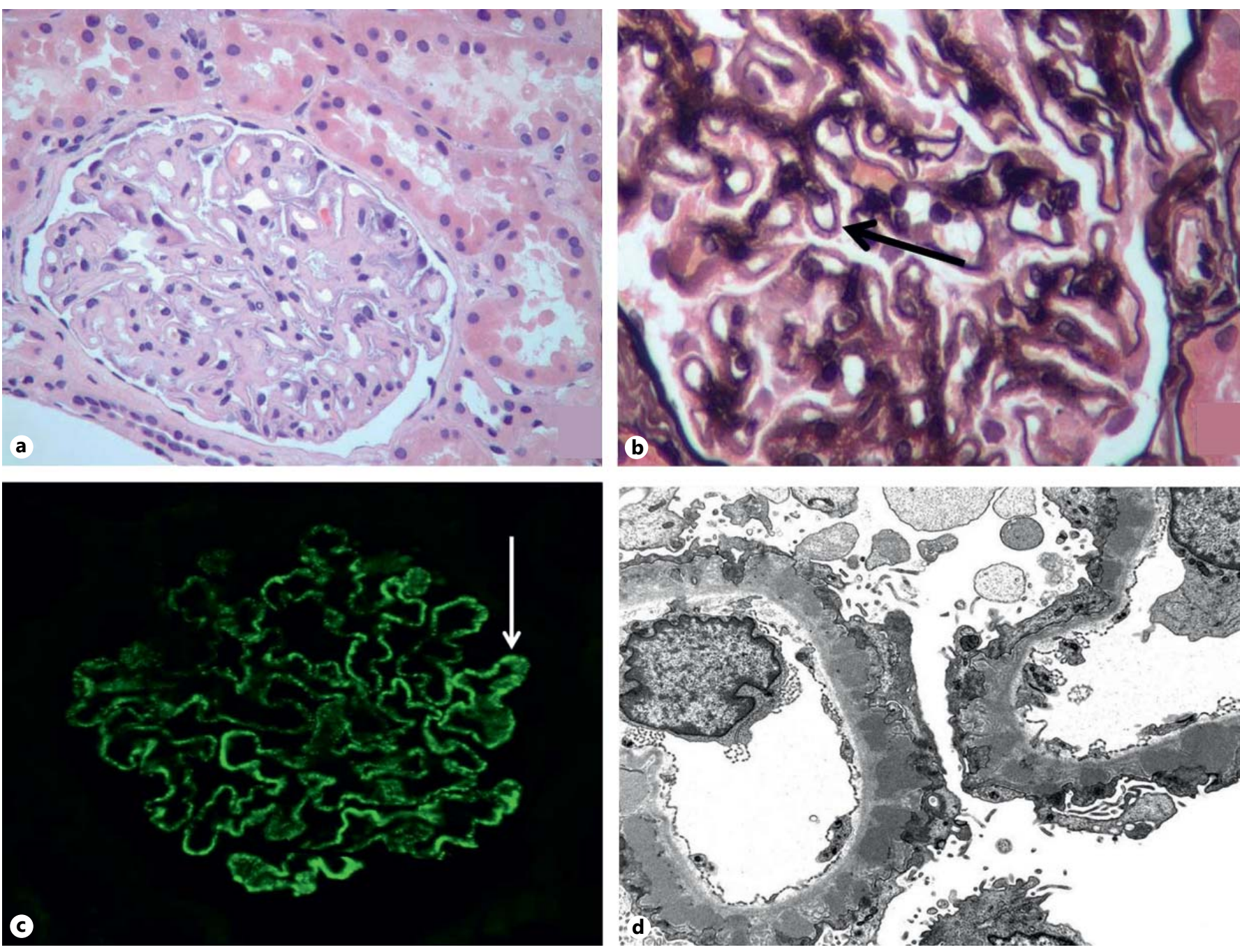

Fig. 1. a Rigid-appearing capillary walls $(H \& E \times 400)$. b Membrane spikes, black arrow (Jones silver satin, $\times 400)$. c Granular capillary loop staining with IgG, white arrow (anti-IgG immunofluorescence, $\times 400$ ). d Subepithelial electron dense deposits (transmission electron microscopy, $\times 6,800$ ).

$[10,15,16,20]$. Therefore, it is important in the workup of a patient with $\mathrm{MN}$ to elucidate the underlying diagnosis as idiopathic or secondary to guide treatment [16].

The case presented here provides many diagnostic nuances. Although it has been well documented that numerous glomerular diseases other than HIVAN, including $\mathrm{MN}$, exist in patients with HIV, these associations have not been very well studied. Most cases of HIV-associated $\mathrm{MN}$ in literature have been reported generally without being specified as idiopathic or secondary $[1,5-9]$. Due to common risk factors, patients with HIV are commonly coinfected with hepatitis $B$, hepatitis $C$, and syphilis, which are associated with secondary $\mathrm{MN}[17,18]$. Furthermore, patients with glomerular disease other than
HIVAN are more likely to be coinfected with hepatitis B or hepatitis C [1].

Multiple reports of HIV-positive patients with MN demonstrated that they responded well to treatment of an underlying etiology consistent with secondary disease. Aydin et al. [21] reported a case of $\mathrm{MN}$ in a patient with recently diagnosed HIV without coinfections whose proteinuria decreased following treatment with ACE and HAART, indicating that MN may have been secondary to HIV. Alarcón-Zurita et al. [22] reported a similar case of $\mathrm{MN}$ in a patient positive for HIV and hepatitis $\mathrm{C}$ whose kidney disease responded to HAART. Chen et al. [23] reported a case of MN in a patient with HIV who was diagnosed with syphilis and who responded positively to 


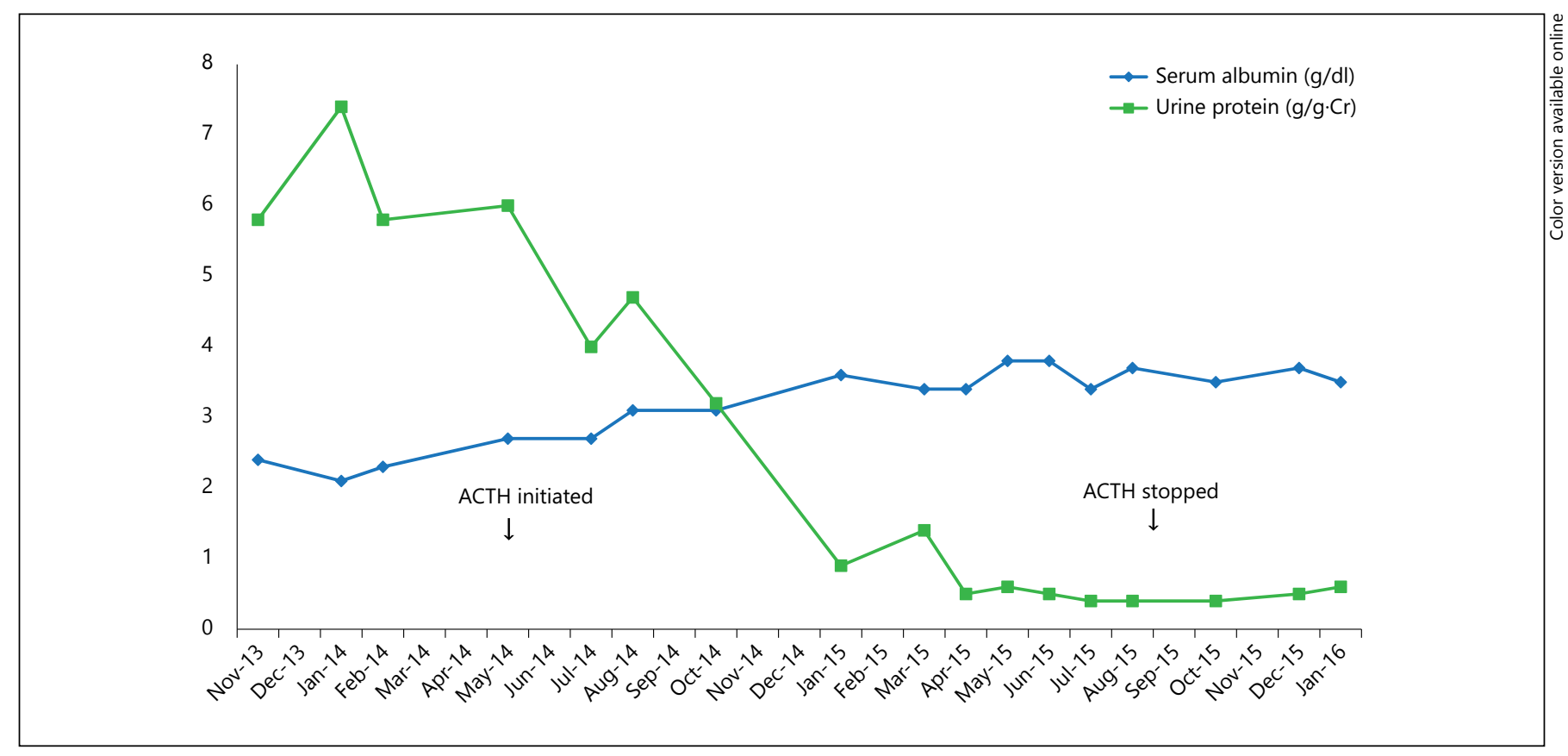

Fig. 2. Serum albumin and random UP/C ratio during the follow-up period.

renal disease when treated with penicillin. Lopez-Lopez et al. [24] identified a patient diagnosed with HIV and systemic lupus erythematosus with $\mathrm{MN}$ that improved after the administration of hydroxychloroquine and HAART.

Unlike these cases, our patient's workup did not reveal any underlying etiology for secondary MN. Our patient's HIV infection was extremely well controlled on HAART with normal CD4 counts and an undetectable viral load, which points away from a connection between his infection and his renal disease. Likewise, our patient's $\mathrm{MN}$ is likely unrelated to his history of hepatitis $C$ infection due to negative tests for hepatitis $C$ by PCR dating back more than 1 year prior to MN diagnosis. Moreover, our patient tested positive for PLA2R antibodies, which has been identified as a sensitive and specific marker associated with idiopathic MN [11]. While anti-PLA2R antibodies have been identified in patients with secondary $\mathrm{MN}$ related to lupus, hepatitis $\mathrm{B}$ and cancer, the prevalence of anti-PLA2R antibodies in these patient groups is low [12]. Testing for anti-PLA2R antibodies has been demonstrated as effective in differentiating idiopathic from secondary $\mathrm{MN}$ with a specificity of $89 \%$ [12-14]. Thus, it is evident that this patient represents a case of idiopathic $\mathrm{MN}$ in a patient with HIV. A blotting technique was used because western blot is the most sensitive assay for the detection of low levels of anti-PLA2R antibody [11]. Both commercially available and easy-to-perform immunofluorescence and ELISA methods would have likely resulted in a negative or ambiguous staining.

In an epidemiologic biopsy study of patients with HIV and renal disease, Szczech et al. [1] described HIV-positive patients with $\mathrm{MN}$ benefitting primarily from treatments other than HAART. Although these findings are consistent with other case reports and with our present case, they are in contrast to the cases mentioned earlier in which renal disease with $\mathrm{MN}$ was effectively treated with HAART $[21,23,25,26]$. It is likely that these conflicting reports represent patients with different subtypes of MN. Renal disease responding to HAART therapy may signify instances of MN secondary to HIV. On the other hand, renal disease not responding to HAART but effectively treated with immunosuppression likely represents idiopathic MN that has developed in a patient infected with HIV. This thought is consistent with our current case of idiopathic $\mathrm{MN}$ as identified by positive anti-PLA2R antibodies that went into remission following immunosuppression as opposed to HAART.

Immune suppression via corticosteroids, cyclosporine, and alkylating agents is generally required for $\mathrm{MN}$ patients with high-grade proteinuria [27]. Unfortunately,
68

Am J Nephrol 2016;43:65-70 DOI: $10.1159 / 000444593$
El-Husseini/Saxon/Jennings/Cornea/ Beck/Sawaya 


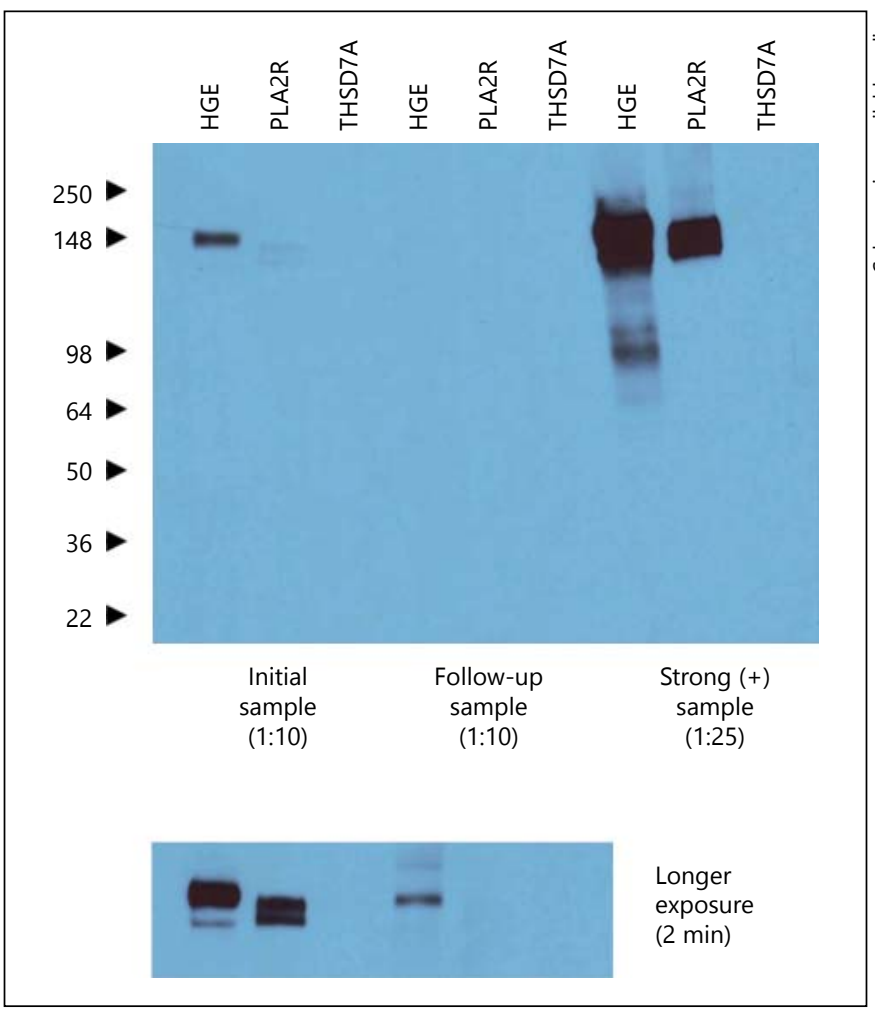

Fig. 3. Western blot of HGE and extracts of cells transfected with human PLA2R or THSD7A were blotted with serum from the patient at a dilution of 1:10. The initial sample shows weak reactivity for native HGE and recombinant PLA2R. Insignificant amount of reactivity was detected in his follow-up sample (lower). In contrast, western blotting with a serum from a patient with primary $\mathrm{MN}$ with high anti-PLA2R titer shows strong signals (top). None of these sera reacted with the THSD7A autoantigen.

such therapies can be associated with significant toxicity, and many patients experience recurrence or develop resistance to treatment [28]. Acthar gel is obtained from the processing of the porcine pituitary gland and is currently the only Food and Drug Administration (FDA)-approved therapy for the treatment of nephrotic syndrome [29]. Despite the severity of proteinuria in our case, we tried to avoid immunosuppressive medications giving the history of HIV and hepatitis C. We initially used the angiotensinconverting enzyme inhibitor with no significant reduction of proteinuria for more than 6 months. We elected to use Acthar gel, because its safely profile appears to be much better than that of the other immunosuppressive and cytotoxic agents [29-31]. ACTH has been used to treat glomerulonephritis based on its steroidogenic effects via the stimulation of adrenal cortisol production. In addition to this steroidogenic mechanism, there is grow-

Treatment of MN with ACTH in HIV

Patient ing evidence that some renoprotective and antiproteinuric consequences of ACTH are related to steroid-independent melanocortin effects on extra-adrenal tissue, including the kidneys. These nonsteroidal results include anti-inflammatory, anti-apoptotic, and immunomodulatory effects that are important in treating glomerular disease $[32,33]$. These mechanisms could help explain the therapeutic benefit of ACTH for our patient without producing significant immunosuppression and activating his underlying infections. One limitation in the present case is that the patient's cortisol levels were not tested before or during ACTH therapy, which may have supported whether his observed remission was more related to steroidogenic or melanocortin effects of therapy.

It cannot be ruled out that the patient's improvement in this case may have represented spontaneous remission of disease given that his improvement occurred within 2 years of diagnosis when most spontaneous remission occurs [19]. However, there was no change in the patient's proteinuria for over 6 months prior to the initiation of ACTH followed by a significant decline in proteinuria in the months following the initiation of ACTH (fig. 2) indicating that remission was more likely related to therapy. The weekly positive serum anti-PLA2R with strongly positive anti-glomerular extracts could suggest the possible coexistence of autoantibodies other than anti-PLA2R. But the relative strength of the PLA2R band between the HGE lane and the recombinant PLA2R lane is more likely due to the relative amounts of the protein in each preparation. Note that the strong positive control also has a stronger band in glomerular extract vs. the recombinant PLA2R lane. The 2 molecules (native vs. recombinant PLA2R) are slightly different, as evidenced by the faster mobility of the recombinant band, which reflects under-glycosylation of the recombinant, cell-expressed band (a common problem with cell-expressed proteins). We know that a given anti-PLA2R-positive serum reacts less well with PLA2R that has been de-glycosylated [11], and thus it is possible that the anti-PLA2R antibodies react less well with the under-glycosylated, cell-expressed PLA2R as well. Although there may be other, unrecognized antigens in the glomerular extract, they would need to run at approximately the same molecular weight to yield such a pattern.

In conclusion, with advancements in HAART and improved control of HIV, cases of glomerular disease other than HIVAN, are likely to be seen more frequently in the HIV-positive population, and MN should be considered in such patients [8]. Patients with $\mathrm{MN}$ who have an apparent underlying cause of secondary MN may benefit from 
further diagnostic efforts to differentiate secondary from idiopathic etiologies. Testing for anti-PLA2R antibodies might help to identify cases of idiopathic $\mathrm{MN}$ that will require immunosuppression. Acthar gel might be useful in the management of complex cases of idiopathic MN.

\section{Disclosure Statement}

None.

\section{References}

1 Szczech LA, Gupta SK, Habash R, Guasch A, Kalayjian R, Appel R, Fields TA, Svetkey LP, Flanagan KH, Klotman PE, Winston JA: The clinical epidemiology and course of the spectrum of renal diseases associated with HIV infection. Kidney Int 2004;66:1145-1152.

2 D'Agati V, Appel GB: HIV infection and the kidney. J Am Soc Nephrol 1997;8:138-152.

3 Wyatt CM, Klotman PE, D'Agati VD: HIVassociated nephropathy: clinical presentation, pathology, and epidemiology in the era of antiretroviral therapy. Semin Nephrol 2008;28:513-522.

4 Klotman PE: HIV-associated nephropathy. Kidney Int 1999;56:1161-1176.

5 Vali PS, Ismal K, Gowrishankar S, Sahay M: Renal disease in human immunodeficiency virus - not just HIV-associated nephropathy. Indian J Nephrol 2012;22:98-102.

6 Nebuloni M, Barbiano di Belgiojoso G, Genderini A, Tosoni A, Riani LN, et al: Glomerular lesions in HIV-positive patients: a 20-year biopsy experience from Northern Italy. Clin Nephrol 2009;72:38-45.

7 George E, Nadkarni GN, Estrella MM, Lucas GM, Sperati CJ, Atta MG, et al: The impact of hepatitis $\mathrm{C}$ coinfection on kidney disease related to human immunodeficiency virus (HIV): a biopsy study. Medicine (Baltimore) 2011;90:289-295.

8 Berliner AR, Fine DM, Lucas GM, Rahman MH, Racusen LC, Scheel PJ, Atta MG: Observations on a cohort of HIV-infected patients undergoing native renal biopsy. Am J Nephrol 2008;28:478-486.

9 D’Agati V, Appel GB: Renal pathology of human immunodeficiency virus infection. Semin Nephrol 1998;18:406-421.

10 Ponticelli C, Glassock RJ: Glomerular diseases: membranous nephropathy - a modern view. Clin J Am Soc Nephrol 2014;9:609616.

11 Beck LH Jr, Bonegio RG, Lambeau G, Beck DM, Powell DW, Cummins TD, Klein JB, Salant DJ: M-type phospholipase A2 receptor as target antigen in idiopathic membranous nephropathy. N Engl J Med 2009;361:11-21.
12 Qin W, Beck LH Jr, Zeng C, Chen Z, Li S, Zuo K, Salant DJ, Liu Z: Anti-phospholipase A2 receptor antibody in membranous nephropathy. J Am Soc Nephrol 2011;22: 1137-1143.

13 Hoxha E, Kneißler U, Stege G, Zahner G, Thiele I, Panzer U, Harendza S, Helmchen UM, Stahl RA: Enhanced expression of the Mtype phospholipase A2 receptor in glomeruli correlates with serum receptor antibodies in primary membranous nephropathy. Kidney Int 2012;82:797-804.

14 VanBeek C, Haas M: Anti-PLA2R-associated membranous nephropathy: a review with emphasis on diagnostic testing methods. Clin Nephrol 2015;84:1-9.

15 Lai WL, Yeh TH, Chen PM, Chan CK, Chiang WC, Chen YM, Wu KD, Tsai TJ: Membranous nephropathy: a review on the pathogenesis, diagnosis, and treatment. J Formos Med Assoc 2015;114:102-111.

16 KDIGO Glomerulonephritis Work Group: KDIGO clinical practice guideline for glomerulonephritis. Kidney Int 2012;2:186-197.

17 Glassock RJ: The pathogenesis of membranous nephropathy: evolution and revolution. Curr Opin Nephrol Hypertens 2012;21:235242.

18 Markowitz GS: Membranous glomerulopathy: emphasis on secondary forms and disease variants. Adv Anat Pathol 2001;8:119-125.

19 Polanco N, Gutiérrez E, Covarsí A, et al: Spontaneous remission of nephrotic syndrome in idiopathic membranous nephropathy. J Am Soc Nephrol 2010;21:697-704.

20 Hofstra JM, Fervenza FC, Wetzels JF: Treatment of idiopathic membranous nephropathy. Nat Rev Nephrol 2013;9:443-458.

21 Aydin S, Mete B, Yilmaz M, Yenidunya G, Zaras R, Tunckale A, Tabak F: A patient with HIV infection presenting with diffuse membranous glomerulonephritis in a country with a low HIV prevalence - remarkable remission with therapy. J Infect Public Health 2012;5: 207-210.

22 Alarcón-Zurita A, Salas A, Antón E, Morey A, Munar MA, Losada P, Martinez J: Membranous glomerulonephritis with nephrotic syndrome in a HIV positive patient - remarkable remission with triple therapy. Nephrol Dial Transplant 2000;15:1097-1098.
23 Chen YM, Marcos LA, Liapis H, Steinberg TH, Morrison AR: An unusual cause of membranous glomerulonephritis in a patient with HIV. Int Urol Nephrol 2012;44:983-986.

24 Lopez-Lopez L, Gonzalez A, Vila LM: Longterm membranous glomerulonephritis as the presenting manifestation of systemic lupus erythematosus in a patient with human immunodeficiency virus infection. Lupus 2012; 21:900-904.

25 Mattana J, Siegal FP, Schwarzwald E, Molho L, Sankaran RT, Gooneratne R, Ahuja TS, Singhal PC: AIDS-associated membranous nephropathy with advanced renal failure: response to prednisone. Am J Kidney Dis 1997;30:116-119.

26 Paueksakon P, Grewal M, Shappell S: Nephrotic range proteinuria and hematuria in a white bisexual male. Am J Kidney Dis 1999; 33:607-612.

27 Cattran D: Management of membranous nephropathy: when and what for treatment. J Am Soc Nephrol 2005;16:1188-1194.

28 Alexopoulos E, Papagianni A, Tsamelashvili $\mathrm{M}$, Leontsini M, Memmos D: Induction and long-term treatment with cyclosporine in membranous nephropathy with the nephrotic syndrome. Nephrol Dial Transplant 2006; 21:3127-3132.

29 Bomback AS, Canetta PA, Beck LH Jr, Ayalon R, Radhakrishnan J, Appel GB: Treatment of resistant glomerular diseases with adrenocorticotropic hormone gel: a prospective trial. Am J Nephrol 2012;36:58-67.

30 Bomback AS, Tumlin JA, Baranski J, et al: Treatment of nephrotic syndrome with adrenocorticotropic hormone (ACTH) gel. Drug Des Devel Ther 2011;5:147-153.

31 Hladunewich MA, Cattran D, Beck LH, et al: A pilot study to determine the dose and effectiveness of adrenocorticotrophic hormone (H.P. Acthar ${ }^{\circledR} \mathrm{Gel}$ ) in nephrotic syndrome due to idiopathic membranous nephropathy. Nephrol Dial Transplant 2014;29:1570-1577.

32 Gong R: The renaissance of corticotropin therapy in proteinuric nephropathies. Nat Rev Nephrol 2011;8:122-128.

33 Gong R: Leveraging melanocortin pathways to treat glomerular diseases. Adv Chronic Kidney Dis 2014;21:134-151. 